\title{
The Evolution of Multidrug Resistance in an Isolated Pseudomonas Strain
}

\author{
Allison Grodnick*a, Ashley Fink, "Timothy J. Johnson ${ }^{b c}$, \& David Mitchelk \\ ${ }^{a}$ Department of Biology, College of St. Benedict St. John's University, Collegeville, $M N$ \\ ${ }^{b}$ Department of Veterinary and Biomedical Sciences, University of Minnesota, St. Paul, MN \\ 'Mid-Central Research and Outreach Center, University of Minnesota, Willmar, MN \\ bttps:/ / doi.org/10.33697/ ajur.2020.026 \\ Student: aegrodnick@gmail.com* \\ Mentor:dmitchell@csbsju.edu
}

\begin{abstract}
As an unintentional result of the extensive use of antibiotics in healthcare and agriculture, antibiotics have become an increasingly prevalent selective pressure on bacteria. This forces bacteria to evolve and acquire antibiotic-resistant genes or mutations in order to survive. Suppose a bacterial strain acquires resistance to three or more antibiotics. In that case, it is deemed multidrug-resistant (MDR), and it becomes a potentially more serious problem to solve in the context of healthcare. This study aims to evaluate the acquisition of resistance to multiple antibiotic drugs by an initially susceptible isolated bacterium from a Minnesota forest environment. The bacterium was found to be Pseudomonas by 16s rRNA gene sequencing. Three antibiotics, neomycin, ciprofloxacin, and imipenem, each from a different drug class, were selected to see if this isolate could become resistant over time and exposure. The bacterial strain developed resistance to the selected antibiotics through a series of sequential exposures to increasing concentrations of each drug in this order. As determined by a disc susceptibility test, the initial isolate acquired resistance to all three selected antibiotics. Single nucleotide polymorphisms (SNPs) between the original isolate and the final resistant strain were identified. These SNPs suggest that mutations to efflux transporters and antibiotic protein targets play a role in acquiring and maintaining antibiotic resistance.
\end{abstract}

\section{KEYWORDS}

Multidrug Resistance; Antibiotics; Neomycin; Ciprofloxacin; Imipenem; Pseudomonas; Evolution; MDR; Minnesota Environment

\section{INTRODUCTION}

Like all species, bacteria evolve. Depending on the environmental factors and stresses, bacteria evolve in order to survive. These factors can range from a change in the environment to human influence. Within the realm of human influence lies antibiotics. While antibiotics are a powerful tool in medicine, they also present a new environmental stressor to bacteria. ${ }^{1}$ As an unintentional result of healthcare and agriculture uses, antibiotics have become an increasingly prevalent selective pressure. $^{2}$ This stressor promotes a bacterium's evolution to become resistant to the antibiotic it is exposed to survive. Those that evolve are then capable of out-competing susceptible bacterial strains, so the resistant strain reproduces more quickly and in greater quantity. When this occurs, it can be difficult to treat patients infected with the now resistant bacteria. This has become a rising health issue as bacteria continue to develop antibiotic resistance; they were previously susceptible. Drug resistance has arisen in some capacity for almost all antibiotic drugs on the market, and certain pathogenic bacterial strains have been characterized as multidrug-resistant organisms. ${ }^{3}$

Antibiotics play an essential role in modern medicine. The average life expectancy before the discovery of antibiotics was 47 years old. ${ }^{4}$ This average life expectancy rose to 78 years following 35 years of antibiotic discovery due to the newly found ability to treat a multitude of infectious diseases. ${ }^{4}$ This resulted in drastic public health changes that improved both quality and length of life. While antibiotics are an essential tool in medicine, problems have begun to arise due to the overuse of antibiotics, leading to antibiotic resistance in bacteria and other microorganisms. The World Health Organization has deemed antibiotic resistance a major public health threat. ${ }^{5}$ The CDC reported antibiotic-resistant bacteria and fungi to have caused at least 2.8 million infections in 2019 in the United States. ${ }^{6}$ Of these cases, 35,000 led to death. Not only does antibiotic resistance affect public health, but it has a negative economic impact too. In the United States, antibiotic-resistant infections are estimated to cost 20 billion dollars per year. ${ }^{5}$ Due to both the health and economic impacts of drug resistance, this problem must be addressed.

Antibiotic drugs fall into four different classes based on their mechanism of action: cell wall inhibitors, protein synthesis inhibitors, DNA and RNA synthesis inhibitors, and metabolic pathway inhibitors. ${ }^{2}$ Not only can bacteria evolve resistance to one 
antibiotic or a class of antibiotics, but they are capable of evolving resistance to multiple drugs and multiple drug classes. The acquisition of resistance to antibiotics holds more serious consequences as a bacterium develops resistance to multiple classes of antibiotics. The term multidrug resistance (MDR) is given to a bacterial strain resistant to a minimum of three different classes of antibiotics. ${ }^{7}$ Bacteria are resistant to antibiotics, either intrinsically or through acquired mutations. ${ }^{7}$ Common mechanisms of resistance include efflux pumps, changes in cell wall permeability, modification of an antibiotic by enzymatic action, antibiotic degradation, use of alternative metabolic pathways, modification of the antibiotic target, and overexpression of the enzyme targeted by an antibiotic. ${ }^{8}$ Efflux pumps have been found to play a pivotal role in both intrinsic and adaptive resistance. Efflux pumps are components of bacterial membranes, so bacteria naturally express these pumps. ${ }^{9}$ In the presence of antibiotic selective pressure, the overexpression of efflux pumps as well as mutations in these pumps have been observed, which has led to efflux pumps becoming a point of interest in the study of antibiotic resistance. ${ }^{9}$

In this study, a bacterium was isolated from the St. John's University arboretum; 16s rRNA gene sequencing determined it to be a Psendomonas. Psendomonas are known to innately express various efflux pumps playing a role in their intrinsic antibiotic-resistant abilities. ${ }^{9}$ The isolate was intrinsically susceptible to an array of antibiotics by antibiotic susceptibility disc tests in which susceptibility is measured by the zone of inhibition created. Three of these antibiotics were selected to evaluate the isolate's ability to acquire MDR: neomycin, ciprofloxacin, and imipenem.

This isolate set the stage for this study's experiment to see if antibiotic resistance could be evolved in the laboratory via repeated exposures to a particular antibiotic. Once resistance was demonstrated via an antibiotic disc test that previously killed the isolate, the isolate's genome was sequenced to look for possible SNPs that arose that may play a role in the acquisition of resistance. The goal was to gain insight into the acquisition of antibiotic resistance in an isolate from an environmental setting rather than with a known pathogenic bacterial strain as other studies have demonstrated.4,7

There are a variety of commonly mutated proteins in resistant Pseudomonas species. ${ }^{4}$ Depending on the selective pressure of a given antibiotic, different mutations may arise in order for the strain to become resistant since antibiotics are bactericidal through a variety of mechanisms.

Neomycin is an aminoglycoside antibiotic. ${ }^{8}$ It is bactericidal through its inhibition of protein synthesis. ${ }^{8}$ Its mechanism of action lies in binding to the $30 \mathrm{~s}$ subunit that is part of the bacterial ribosome. ${ }^{10}$ Due to this binding action, the ribosome cannot properly translate mRNA into protein, which is detrimental to the cell..$^{10}$ In order to survive, bacteria have developed mechanisms of resistance to this class of antibiotics. ${ }^{8}$ A variety of mechanisms have been identified in the gaining of resistance to aminoglycosides: active efflux, permeability changes, ribosome modifications, and degradation of aminoglycosides by enzymatic activity. ${ }^{8}$ These changes are attributed to a variety of genetic mutations, so resistance to this antibiotic originates from a variety of genes. ${ }^{8}$

Ciprofloxacin belongs to the DNA synthesis inhibitor drug class. It is a fluoroquinolone. ${ }^{8}$ It works by inhibiting DNA gyrase and topoisomerase IV. ${ }^{8}$ Without these enzymes, the bacterium cannot replicate its DNA, which is an essential part of the growth of a bacterial population. ${ }^{8}$ In order to gain resistance, a bacterium typically gains a random mutation in one of the genes that codes for these targeted enzymes. ${ }^{8}$ A random mutation in a targeted enzyme may then alter the enzyme's structure, which may lead to altered enzyme function. ${ }^{8}$ Resistant bacteria have also been observed to decrease membrane permeability and perform active efflux in order to gain resistance to this class of drugs. ${ }^{8}$

Imipenem falls into the cell wall inhibitor drug class. It is a carbapenem that belongs to a group of antibiotics called $\beta$-lactams. ${ }^{8}$ These antibiotics work by weakening the cell wall of a bacterium and making it more susceptible to lysing due to osmotic pressure. ${ }^{8}$ It works by binding penicillin-binding proteins, which play a role in the cross-linking of peptidoglycan chains, which are structurally needed in the cell wall. ${ }^{8}$ Many bacteria have successfully acquired resistance to drugs in this class through the expression of beta-lactamase, which destroys the beta-lactam ring in these drugs. ${ }^{8} \mathrm{~A}$ variety of other mechanisms to resistance have been observed, but beta-lactamase production is the most common. ${ }^{8}$

Taken together, these selected antibiotics target bacteria cells using very different and distinct mechanisms. The isolated Pseudomonas was evaluated for its ability to gain and maintain resistance to each of these antibiotics. This study aims to provide insight into this acquisition and maintenance of multidrug resistance in a bacterial strain isolated from an environmental setting.

\section{METHODS AND PROCEDURES}

\section{Materials}

Tryptic soy agar (TSA) and tryptic soy broth (TSB) were used for growing bacteria on plates and in liquid cultures, respectively (BD). The TSB flasks were incubated at $25^{\circ} \mathrm{C}$ and $200 \mathrm{rpm}$ (New Brunswick Scientific C25 Incubator Shaker) for 24 to 72 hours. 
Optical density at $600 \mathrm{~nm}$ was taken to measure culture density ( $\mathrm{OD}_{600}$; Beckman DU640 spectrophotometer). Neomycin trisulfate salt hydrate (Sigma Aldrich, CAS 1405-10-3), ciprofloxacin (Sigma Aldrich, CAS 85721-33-1), and imipenem monohydrate (Sigma Aldrich, CAS 74431-23-5) powders were used for the series of sequential exposures. $30 \mu \mathrm{g} / \mathrm{mL}$ neomycin discs (BD BBL, 231313), $5 \mu \mathrm{g} / \mathrm{mL}$ ciprofloxacin discs (BD BBL, 231658), and $10 \mu \mathrm{g} / \mathrm{mL}$ imipenem discs (BD BBL, 231645) were used in the antibiotic susceptibility disc tests.

Colony isolation and $r \mathrm{RN} A$ gene sequencing identification

Water samples from a pond located in the St. John's University Arboretum in Collegeville, MN, were collected and streaked out on a TSA plate. A single colony was selected and isolated by line streaking until pure. A pure culture of the isolated bacteria was subject to PCR amplification using universal primers U341F and UA1406R that recognize an 1100 bp segment of the 16s rRNA gene. PCR products were run on a 1.5\% agarose gel and confirmed to be $1100 \mathrm{bp}$ in length. PCR products were then purified (QIAquick PCR Purification Kit, QIAGEN), pre-mixed with the forward universal primer U341F, and sent to GeneWiz (South Plainfield, NJ) for sequencing. FASTA files were then used in a BLAST and the ARB-SLIVA project aligner to identify each organism's most likely genus and species.

\section{Antibiotic exposure}

The isolate was determined to be susceptible to neomycin $(30 \mu \mathrm{g} / \mathrm{mL})$, ciprofloxacin $(5 \mu \mathrm{g} / \mathrm{mL})$, and imipenem $(10 \mu \mathrm{g} / \mathrm{mL})$ by an antibiotic susceptibility disc test. The zone of inhibition (measured in millimeters) created by the disc was used as a means of measuring susceptibility to the given antibiotic.

A flask containing $50 \mathrm{~mL}$ of TSB with a concentration of $5 \mu \mathrm{g} / \mathrm{mL}$ of neomycin was inoculated with the original Pseudomonas isolate at time $0 \mathrm{hr}$. The optical density (OD600) was measured every two hours until an absorbance of 1.0 was reached after 24 to 72 hours. Once achieved, the evolved bacteria were streaked out onto a TSA plate without the antibiotic present. This process was repeated with the evolved bacterial strain at a higher concentration of neomycin until the strain acquired resistance to neomycin at a concentration of $30 \mu \mathrm{g} / \mathrm{mL}$. The bacterial strain developed resistance to neomycin at the increments of $5 \mu \mathrm{g} / \mathrm{mL}$, $7.5 \mu \mathrm{g} / \mathrm{mL}, 15 \mu \mathrm{g} / \mathrm{mL}, 20 \mu \mathrm{g} / \mathrm{mL}, 25 \mu \mathrm{g} / \mathrm{mL}$, and $30 \mu \mathrm{g} / \mathrm{mL}$ in sequential order. There were a total of six transfers within this series in which the isolate was streaked for isolation before each transfer. To confirm the acquisition of resistance, the bacterial strain at each neomycin concentration increment was plated on a TSA plate and tested against the $30 \mu \mathrm{g} / \mathrm{mL}$ neomycin disc. Zones of inhibition were measured after a 48-hour growth period.

Once the acquisition of antibiotic resistance to neomycin at the target concentration of $30 \mu \mathrm{g} / \mathrm{mL}$ was confirmed by the disc test, the resistant strain underwent a process of sequential exposure again but with ciprofloxacin. The strain developed resistance to ciprofloxacin at the increments $2.5 \mu \mathrm{g} / \mathrm{mL}, 3.5 \mu \mathrm{g} / \mathrm{mL}, 4.5 \mu \mathrm{g} / \mathrm{mL}$, and $5 \mu \mathrm{g} / \mathrm{mL}$. There were a total of four transfers within this series, and colonies were streaked for isolation before each new transfer. In order to confirm the acquisition of resistance, the bacterial strain at each ciprofloxacin concentration increment was plated on a TSA plate and tested against the $5 \mu \mathrm{g} / \mathrm{mL}$ ciprofloxacin disc. Zones of inhibition were measured after a 48-hour growth period.

This process of sequential exposure was repeated once more with imipenem. The initial bacterial strain used for this round of sequential exposure was the strain previously made resistant to $30 \mu \mathrm{g} / \mathrm{mL}$ neomycin and $5 \mu \mathrm{g} / \mathrm{mL}$ ciprofloxacin. The bacterial strain developed resistance to imipenem at the increments $1 \mu \mathrm{g} / \mathrm{mL}, 3 \mu \mathrm{g} / \mathrm{mL}, 5 \mu \mathrm{g} / \mathrm{mL}, 7.5 \mu \mathrm{g} / \mathrm{mL}$, and $10 \mu \mathrm{g} / \mathrm{mL}$. There were a total of five transfers in this series, and each isolate was streaked for isolation before a new transfer. Following the acquisition of antibiotic resistance to imipenem at the target concentration of $10 \mu \mathrm{g} / \mathrm{mL}$, the bacterial strain at each imipenem concentration increment was plated on a TSA plate and tested against the $10 \mu \mathrm{g} / \mathrm{mL}$ ciprofloxacin disc to confirm that resistance to imipenem was acquired. Zones of inhibition were measured after a 48-hour growth period.

Throughout this series of sequential exposures, there were a total of 15 transfers of the bacterial strain to an increased antibiotic concentration. In the neomycin experiment set, there were six transfers. In the ciprofloxacin experiment set, there were five transfers. Lastly, in the imipenem experiment set, there were four transfers.

\section{Antibiotic susceptibility disc test for $M D R$}

TSA plates containing $30 \mu \mathrm{g} / \mathrm{mL}$ neomycin and $10 \mu \mathrm{g} / \mathrm{mL}$ imipenem were made. The bacterial strain at each major point in the study was plated onto these antibiotic TSA plates, and a $5 \mu \mathrm{g} / \mathrm{mL}$ ciprofloxacin disc was used. The strains used were the initial isolate, the $30 \mu \mathrm{g} / \mathrm{mL}$ neomycin resistant strain, the $30 \mu \mathrm{g} / \mathrm{mL}$ neomycin and $5 \mu \mathrm{g} / \mathrm{mL}$ ciprofloxacin-resistant strain, and the 30 $\mu \mathrm{g} / \mathrm{mL}$ neomycin, $5 \mu \mathrm{g} / \mathrm{mL}$ ciprofloxacin, and $10 \mu \mathrm{g} / \mathrm{mL}$ imipenem resistant strain. Zones of inhibition were measured after a 
48-hour growth period. Refer to Table 1 for the standard zone of inhibition measurements used to determine whether or not a strain was resistant.

Genome Sequencing

The original isolate's genome, the neomycin resistant strain, the neomycin, and ciprofloxacin-resistant strain, and the neomycin, ciprofloxacin, and imipenem resistant strain were sequenced and analyzed by Illumina sequencing. Flask growths were grown overnight on MacConkey agar to check for visible contamination, then a streak of colonies from the plate was re-grown overnight in $2 \mathrm{~mL} \mathrm{LB}$ broth with shaking at $37^{\circ} \mathrm{C}$. DNA was extracted from overnight growths of each strain using the Qiagen DNEasy Kit. Library preparations were performed with Illumina reagents (Nextera XT DNA library kit). DNA sequencing was performed on a MiSeq machine at the University of Minnesota Genomics Center (Saint Paul, MN, USA). A range of 81,420-666,386 highquality reads (Q30 average) was generated for each strain, corresponding to approximately 10-30-fold coverage for each strain sequenced. Following quality filtering, all genomes were assembled using SPAdes. ${ }^{11}$ The original strain's assembly was used as a reference for read mapping to identify SNPs, with 463 ( $\mathrm{min}=120)$ contigs totaling 6,743,307 bp. The assembly of this genome was annotated using Prokka. ${ }^{12}$ Reads from the original strain and evolved strains were mapped to the original strain assembly using snippy (https://github.com/tseemann/snippy), with a similarity fraction of 0.9 and minimum coverage of $8 x$ for variant calling. Variants were called at high minimum frequency $(>90 \%)$ to identify true variants relative to the reference genome and other sequenced strains in this study. Mutations unique to each strain sequenced, relative to the parent strain sequenced, were identified and annotated. MAUVE. ${ }^{13}$ was used to align the original and evolved sequences for the assessment of any large-scale genomic changes.

\section{RESULTS AND DISCUSSION}

Antibiotic Exposure and Disc Test

The basis of the experiment is to evaluate the ability of a bacterial strain to gain antibiotic resistance. The original bacterial isolate is referred to as strain 1.

The optical density at $600 \mathrm{~nm}$ was used to measure the growth of the bacteria. Each flask was grown to a minimum optical density of 1.0. Due to overnight growth in some of the flasks, there were cases where flasks exceeded the 1.0 minimum optical density. A growth curve was made with the average of two replicate flasks' optical densities at each time interval measured for each antibiotic concentration. Additionally, the standard deviation was calculated and applied as error bars. The majority of the standard deviations calculated were small, which is evident from the size of the error bars. The growth curve for the bacterial strain at each neomycin concentration is depicted in Figure 1; the neomycin resistant strain is referred to as "strain 2." Figure 2 depicts the growth curve for the bacterial strain at each ciprofloxacin concentration; this neomycin and ciprofloxacin-resistant strain is referred to as "strain 3." Lastly, Figure 3 depicts the growth curve of the bacterial strain at each imipenem concentration; this final strain is referred to as "strain 4." For clarity, it is worth noting that the original isolated bacterial strain, parental strain, is referred to as "strain 1. ."
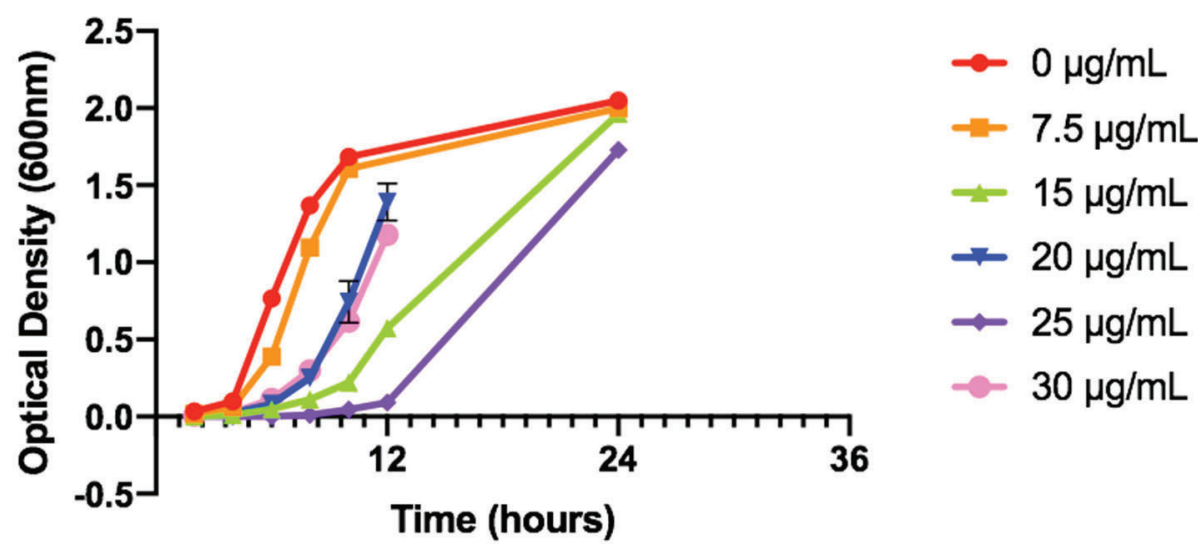

Figure 1. Growth curve for the series sequential exposures to neomycin at each concentration. 


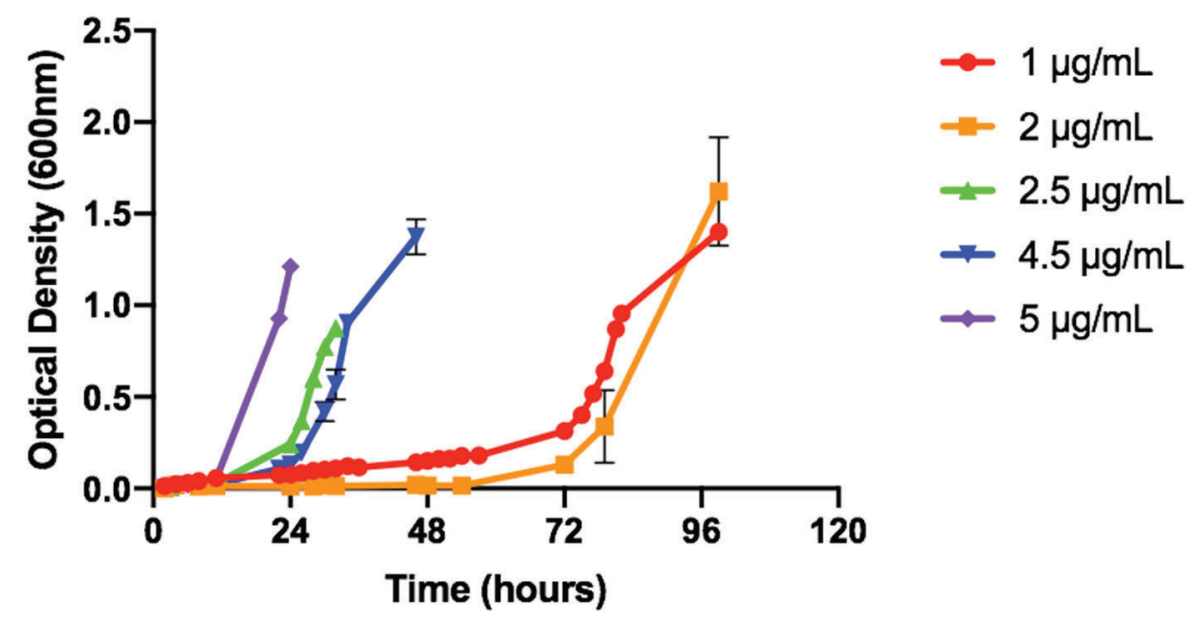

Figure 2. Growth curve for the series sequential exposures to ciprofloxacin at each concentration.

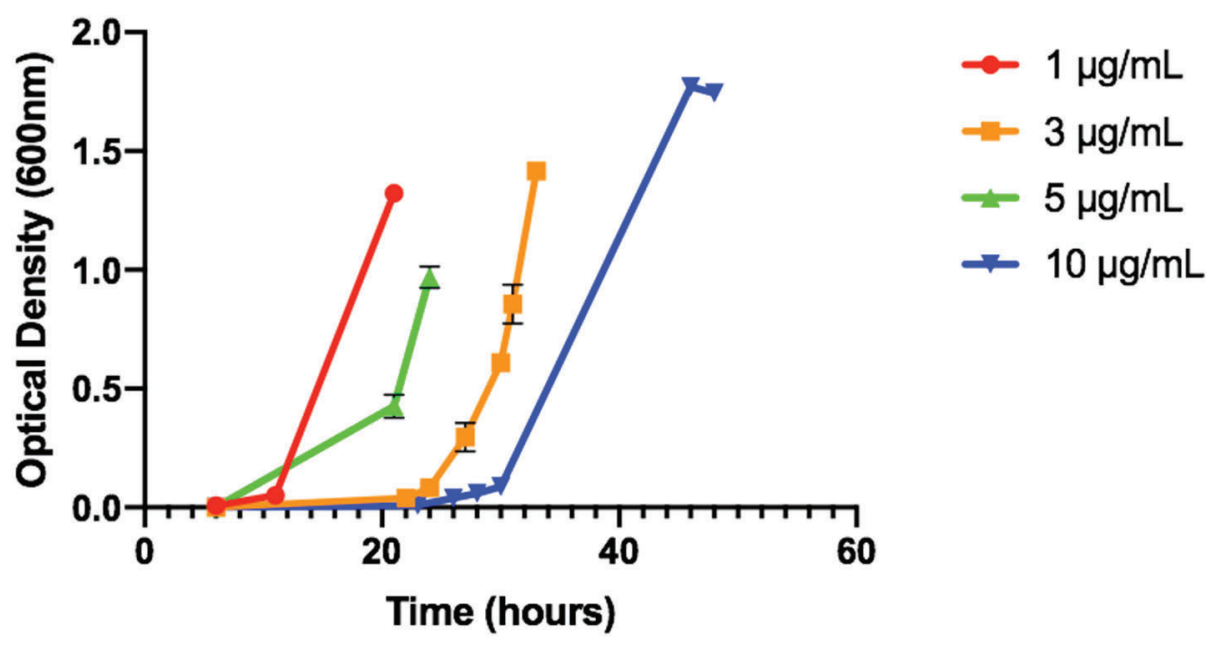

Figure 3. Growth curve for the series sequential exposures to imipenem at each concentration

\begin{tabular}{|l|l|l|l|}
\hline Antibiotic & Resistant & Intermediate & Susceptible \\
\hline Neomycin & $12 \geq$ & $13-16$ & $\geq 17$ \\
\hline Ciprofloxacin & $15 \geq$ & $16-20$ & $\geq 21$ \\
\hline Imipenem & $13 \geq$ & $14-15$ & $\geq 16$ \\
\hline
\end{tabular}

Table 1. Standard zones of inhibition ( $\mathrm{mm}$ ) for each antibiotic according to Becton Dickinson BBL Sensi-Disc Antimicrobial Sensibility Test standards.

\begin{tabular}{|l|l|}
\hline Antibiotic & Initial Zone of Inhibition \\
\hline Neomycin & 20 \\
\hline Ciprofloxacin & Not Recorded \\
\hline Imipenem & 39 \\
\hline
\end{tabular}

Table 2. Initial zones of inhibition (mm) were measured for the isolate prior to the series of sequential exposures. ${ }^{*}$ Initial zone of inhibition was not recorded.

The Pseudomonas was first evolved to acquire resistance to neomycin. The bacterial strain developed resistance to neomycin at the increments of $5 \mu \mathrm{g} / \mathrm{mL}, 7.5 \mu \mathrm{g} / \mathrm{mL}, 15 \mu \mathrm{g} / \mathrm{mL}, 20 \mu \mathrm{g} / \mathrm{mL}, 25 \mu \mathrm{g} / \mathrm{mL}$, and $30 \mu \mathrm{g} / \mathrm{mL}$ in a sequential order. The optical density measured at $600 \mathrm{~nm}$ reached 1.0 for each increment (Figure 1). As seen in Figure 4a, the zone of inhibition began to disappear as the antibiotic concentration increased. This continued until the Pseudomonas was resistant to neomycin at $30 \mu \mathrm{g} / \mathrm{mL}$ in which there was no longer a zone of inhibition (Figure 4a). This shows that the earlier transfers of the bacterial strain were still 
susceptible to the high dose of neomycin since it only gained resistance at a low, sub-lethal dose. This resulted in a relatively large zone of inhibition (Figure 4a). As this sub-lethal dose that the strain could survive increased, the strain became less susceptible to the antibiotic on disc and, therefore, more resistant, as shown with a smaller inhibition zone (Figure 4a, Table 3). Eventually, this pushed the strain to gain resistance to neomycin at the initially susceptible neomycin concentration yielding bacterial strain 2 .

Next, strain 2 was mutated via culture to gain resistance to ciprofloxacin. Strain 2 developed resistance to ciprofloxacin at increments of $2.5 \mu \mathrm{g} / \mathrm{mL}, 3.5 \mu \mathrm{g} / \mathrm{mL}, 4.5 \mu \mathrm{g} / \mathrm{mL}$, and $5 \mu \mathrm{g} / \mathrm{mL}$. The optical density measured at $600 \mathrm{~nm}$ reached 1.0 for each increment (Figure 2). Figure 4b depicts this series of sequential exposures and shows that the strain was able to gain resistance due to the lack of a zone of inhibition, so it ultimately evolved into strain 3 in which it was resistant to both neomycin and ciprofloxacin. The measurements of each zone can be found in Table 3.

Lastly, strain 3 underwent a series of sequential exposures until it acquired resistance to imipenem at an initially lethal concentration as well. The bacterial strain developed resistance to imipenem at the increments $1 \mu \mathrm{g} / \mathrm{mL}, 3 \mu \mathrm{g} / \mathrm{mL}, 5 \mu \mathrm{g} / \mathrm{mL}, 7.5$ $\mu \mathrm{g} / \mathrm{mL}$, and $10 \mu \mathrm{g} / \mathrm{mL}$. The optical density measured at $600 \mathrm{~nm}$ reached 1.0 for each increment (Figure 3). As the sub-lethal dose strain 3 was able to survive at was increased, the zone of inhibition induced by the imipenem disc was reduced, suggesting that the strain became less susceptible to the antibiotic disc and therefore more resistant to the drug (Figure 4c, Table 3). Eventually, the strain gained resistance to imipenem at the initially lethal imipenem concentration yielding strain 4 . Evidence of the acquisition of imipenem resistance is shown by the lack of a zone of inhibition after the final transfer (Figure 4c).
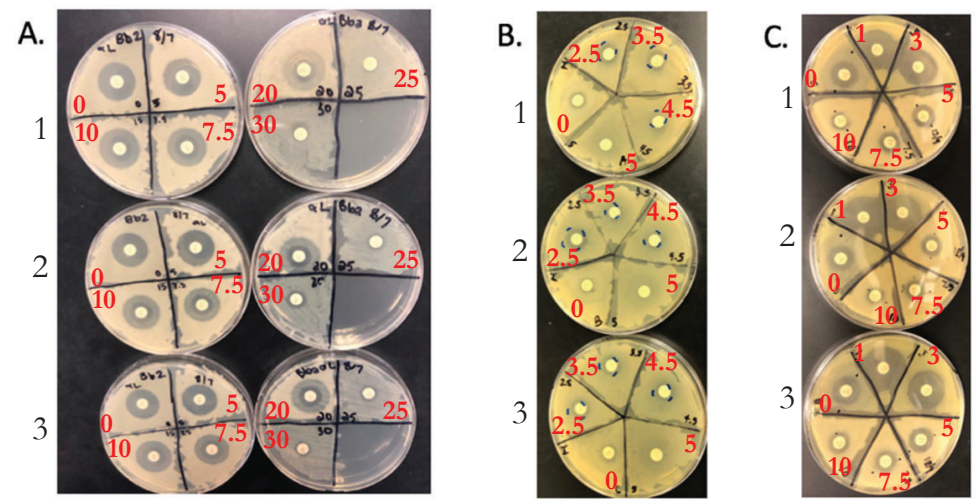

Figure 4. Antibiotic susceptibility disc tests after each series of sequential exposures with each antibiotic. The red number in each pie square is indicative of the antibiotic concentration $(\mu \mathrm{g} / \mathrm{mL})$ in which that strain of bacteria was exposed to and was able to grow. (a) Neomycin; original isolate used for initial inoculation. (b) Ciprofloxacin; neomycin resistant strain $(30 \mu \mathrm{g} / \mathrm{mL})$ used for initial inoculation. The area labeled " $0 \mu \mathrm{g} / \mathrm{mL}$ " indicates that the strain was not exposed to ciprofloxacin, and a neomycin disc was used as a control. (c) Imipenem; neomycin $(30 \mu \mathrm{g} / \mathrm{mL})$ and ciprofloxacin $(5 \mu \mathrm{g} / \mathrm{mL})$ resistant strain used for initial inoculation. The area labeled " $0 \mu \mathrm{g} / \mathrm{mL}$ " indicates that the strain was not exposed to imipenem, and a ciprofloxacin disc was used as a control.

\begin{tabular}{|c|c|c|c|c|c|c|c|c|c|}
\hline \multirow{2}{*}{$\begin{array}{c}\text { [Antibiotic] } \\
\text { Exposure }\end{array}$} & \multicolumn{3}{|c|}{ Neomycin } & \multicolumn{3}{|c|}{ Ciprofloxacin } & \multicolumn{3}{|c|}{ Imipenem } \\
\hline & 1 & 2 & 3 & 1 & 2 & 3 & 1 & 2 & 3 \\
\hline 0 & 20 & 20 & 19 & & & & & & \\
\hline 1 & & & & & & & 26 & 22 & 22 \\
\hline 2.5 & & & & 13 & 14 & 11 & & & \\
\hline 3 & & & & & & & 20 & 20 & 20 \\
\hline 3.5 & & & & 11 & 0 & 11 & & & \\
\hline 4.5 & & & & 8 & 0 & 0 & & & \\
\hline 5 & 20 & 20 & 19 & 0 & 0 & 0 & 15 & 14 & 15 \\
\hline 7.5 & 12 & 14 & 13 & & & & 12 & 12 & 13 \\
\hline 10 & 12 & 13 & 12 & & & & 11 & 9 & 10 \\
\hline 20 & 11 & 12 & 12 & & & & & & \\
\hline 25 & 0 & 0 & 0 & & & & & & \\
\hline 30 & 0 & 0 & 0 & & & & & & \\
\hline
\end{tabular}

Table 3. Zones of inhibition ( $\mathrm{mm}$ ) following antibiotic susceptibility disc tests after each series of sequential exposures with each antibiotic. Boxes shaded grey are not applicable to that antibiotic. Refer to Table 1 to compare to the standards. Bold values indicate resistance gained by the bacterial strain at the initially lethal dose for each drug. 
In order to evaluate whether or not the Psendomonas was able to maintain resistance to each drug, a multidrug susceptibility disc test was performed. Tryptic soy agar plates with $30 \mu \mathrm{g} / \mathrm{mL}$ neomycin and $10 \mu \mathrm{g} / \mathrm{mL}$ imipenem were made. The bacterial strain at each major point in the study was plated onto these antibiotic TSA plates, and a $5 \mu \mathrm{g} / \mathrm{mL}$ ciprofloxacin disc was used. The results of this disc test are shown in Figure 5. This provides evidence that not only was the Pseudomonas able to gain resistance to each antibiotic, but it was able to maintain this resistance over time. Throughout these series of sequential exposures, there were a total of 15 passages of the bacterial strain to an increased antibiotic concentration. In the neomycin experiment set, there were six passages. In the ciprofloxacin experiment set, there were five passages. Lastly, in the imipenem experiment set, there were four passages.

Taken together, the Pseudomonas isolate was forced to sequentially gain and maintain resistance by shaking with increasing concentrations of each antibiotic (Figure 5d). This acquisition of resistance was not unique to a particular drug class but rather included antibiotics from three different drug classes: a protein synthesis inhibitor (neomycin), a DNA synthesis inhibitor (ciprofloxacin), and a cell wall inhibitor (imipenem).
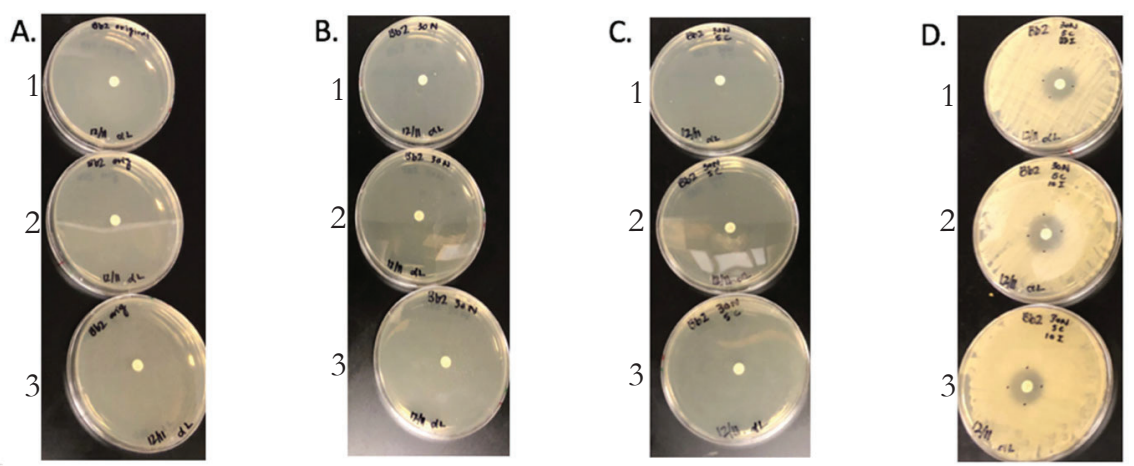

Figure 5. Antibiotic susceptibility disc test ( $5 \mu \mathrm{g} / \mathrm{mL}$ ciprofloxacin disc) on antibiotic TSA plate (neomycin $30 \mu \mathrm{g} / \mathrm{mL}$, imipenem $10 \mu \mathrm{g} / \mathrm{mL}$ ) shows that the original isolate successfully acquired MDR to neomycin, imipenem, and ciprofloxacin. (a) Triplicates with strain 1. (b) Triplicates with strain 2. (c) Triplicates with strain 3. (d) Triplicates with strain 4.

\section{Genome Sequencing}

After the bacterial strain successfully gained resistance to each antibiotic, the genomes of four strains were sequenced and analyzed for SNPs and other genomic changes. MAUVE was used to align genome assemblies for the original and evolved strains in this study. These strains included the original isolate (strain 1), the neomycin resistant strain (strain 2), the neomycin and ciprofloxacin-resistant strain (strain 3), and the neomycin, ciprofloxacin, and imipenem resistant strain (strain 4). While other SNPs were generated from the genome sequencing analysis, Table 4 represents SNPs deemed relevant in the study, including SNPs involving efflux transporters or drug targets of each antibiotic. No large scale insertions or deletions were identified. A total of 1066 mutations between the original isolate and the final MDR strain were found. It is worth noting that the annotation of the gene places an underscore followed by a number when multiple alleles of a gene are identified. This is based on $90 \%$ a.a. similarity, so these are predicted proteins similar to the gene listed.

\begin{tabular}{|c|c|c|c|c|c|c|c|c|}
\hline \multirow[t]{2}{*}{ BP Position } & \multicolumn{4}{|c|}{ Strain } & \multirow{2}{*}{ Mutation } & \multirow[t]{2}{*}{ AA Change } & \multirow[t]{2}{*}{ Gene } & \multirow{2}{*}{ Protein Affected } \\
\hline & 1 & 2 & 3 & 4 & & & & \\
\hline 25477 & & $\mathrm{X}$ & $\mathrm{X}$ & $\mathrm{X}$ & $\mathrm{G}$ to $\mathrm{T}$ & Leu 40 to Ile & \multirow[t]{2}{*}{ emrB } & \multirow{2}{*}{$\begin{array}{c}\text { EmrAB-TolC multidrug efflux transport system - } \\
\text { membrane subunit }\end{array}$} \\
\hline 25506 & & $\mathrm{X}$ & $\mathrm{X}$ & $\mathrm{X}$ & $\mathrm{C}$ to $\mathrm{G}$ & Ser 30 to Thr & & \\
\hline 10233 & & $\mathrm{X}$ & $\mathrm{X}$ & $\mathrm{X}$ & C to $\mathrm{G}$ & Ala 27 to Pro & \multirow[t]{4}{*}{$m d t C$} & \multirow{4}{*}{$\begin{array}{l}\text { MdtABC-TolC multidrug efflux transport system - } \\
\text { membrane subunit }\end{array}$} \\
\hline 10238 & & $\mathrm{X}$ & $\mathrm{X}$ & $\mathrm{X}$ & $\mathrm{C}$ to $\mathrm{A}$ & Arg 25 to Leu & & \\
\hline 10279 & & $\mathrm{X}$ & $\mathrm{X}$ & $\mathrm{X}$ & T to $G$ & Lys 11 to Asn & & \\
\hline 10289 & & $\mathrm{X}$ & $\mathrm{X}$ & $\mathrm{X}$ & $\mathrm{C}$ to $\mathrm{A}$ & Gly 8 to Val & & \\
\hline 39721 & & & $\mathrm{X}$ & $\mathrm{X}$ & A to $\mathrm{T}$ & Leu 55 to Gln & gyrA & DNA gyrase, subunit A \\
\hline 99267 & & & $\mathrm{X}$ & $\mathrm{X}$ & A to $\mathrm{C}$ & Glu 469 to Asp & $g y r B$ & DNA gyrase, subunit B \\
\hline 36359 & & & $\mathrm{X}$ & $\mathrm{X}$ & A to $\mathrm{T}$ & Ter 562 to Tyr & parC & Topoisomerase IV subunit A \\
\hline 42508 & & & & $\mathrm{X}$ & $\mathrm{C}$ to $\mathrm{G}$ & Ala 546 to Pro & $m d t C$ & $\begin{array}{c}\text { MdtABC-TolC multidrug efflux transport system - } \\
\text { membrane subunit }\end{array}$ \\
\hline 78781 & & & & $\mathrm{X}$ & $\mathrm{G}$ to $\mathrm{T}$ & Asp 617 to Glu & $\operatorname{mrd} A$ & $\begin{array}{c}\text { Cell shape; peptidoglycan synthetase; penicillin-binding } \\
\text { protein } 2\end{array}$ \\
\hline
\end{tabular}

Table 4. Genome mapping reveals SNPs acquired and maintained in response to each antibiotic stressor. Strain 1 refers to the original isolate. Strain 2 refers to the neomycin resistant strain. Strain 3 refers to the neomycin and ciprofloxacin-resistant strain. Strain 4 refers to the neomycin, ciprofloxacin, and imipenem resistant strain. An "X" indicates that the strain has the corresponding mutation. Under the amino acid change column, "ter" refers to termination. 
While neomycin binds to the 30s subunit of the bacterial ribosome, thereby inhibiting protein synthesis, there were no mutations in the neomycin resistant strain that pertained to the 30s ribosomal subunit (Table 4). However, mutations were seen in the DNA coding for both the EmrAB-TolC multidrug efflux transport system and the MdtABC-TolC multidrug efflux transport systemboth acquired mutations in the membrane subunit of these efflux transporters (Table 4). These transport systems are multiprotein complexes that involve both the inner and outer membranes of gram-negative bacteria. ${ }^{14}$ The TolC portion of the system is embedded in the outer membrane and works in synergy with a variety of active transporters, including the EmrAB or MdtABC transmembrane proteins that lie in the inner membrane. ${ }^{14} \mathrm{TolC}$ acts as a channel that transports molecules out of the periplasm that is pumped in by the active transporters EmrAB or MdtABC. ${ }^{14} \mathrm{TolC}$ is capable of transporting a wide variety of molecules, including antibiotics, antibacterial peptides, bile salts, and toxins. ${ }^{14}$ Since TolC is already versatile; it is more likely that the selective pressure of neomycin resulted in mutations in the EmrAB and MdtABC domains of the efflux transporters. The acquisition of mutations in these genes suggests that the original isolate improved its ability to actively pump antibiotics out of its cell through mutations to these transporters. Active efflux is one of the most common mechanisms of antibiotic resistance, so this mechanism likely played a role in the acquisition of resistance to neomycin while undergoing neomycin as a selective pressure.

The two missense mutations in the EmrAB-TolC transporter resulted in amino acid changes, but the mutations yielded structurally and chemically similar amino acids to the original protein. At the base pair position of 25477 , leucine 40 was exchanged for isoleucine (Table 4). Both of these amino acids are hydrophobic but have subtle structural changes. Similarly, the other missense mutation in this protein complex was serine 30 to threonine (Table 4). Both of these amino acids are polar and slightly different in structure.

Additionally, there were four missense mutations in the MdtABC-TolC multidrug efflux transport system. At the base pair position 10233, alanine 27 was changed to proline. At the base pair position 10238, arginine 25 was changed to leucine. At the base pair position 10279, lysine 11 was changed to asparagine. Lastly, at the base pair position 10289, glycine 8 was changed to valine. (Table 4) The effect of these mutations on protein structure is unclear, but due to the efflux transport system's function, they may have improved the abilities of the efflux transport system to pump neomycin out of the cell.

After gaining resistance to neomycin through point mutations, the strain further mutated under the selective pressure of ciprofloxacin until it gained ciprofloxacin resistance. Strain 3 maintained the SNPs in the EmrAB-TolC multidrug efflux transport system and the MdtABC-TolC multidrug efflux transport system acquired by strain 2 under the neomycin selective pressure (Table 4). In addition to maintaining these mutations, the strain acquired SNPs in the genes encoding for DNA gyrase and topoisomerase IV. These are likely significant mutations since ciprofloxacin directly targets both of these enzymes in order to kill the bacterial cell. Ciprofloxacin is able to inhibit DNA replication through its inhibition of these proteins.

Ciprofloxacin resistance in bacteria is attributed to an increase in efflux transporter expression and mutations of the enzymes DNA gyrase and topoisomerase IV that lower the drug's binding affinity to these enzymes. ${ }^{12}$ SNPs in both DNA gyrase and topoisomerase IV were found (Table 4). There was a mutation to the DNA gyrase subunit B in which glutamic acid 469 was changed to aspartic acid. This mutation has been found to be a key mutation in the lowering of the binding affinity of ciprofloxacin to DNA gyrase in other studies that have evaluated the acquisition of ciprofloxacin resistance in Psendomonas aeruginosa.$^{15}$ Additionally, leucine 55 was found to be mutated to glutamine in DNA gyrase subunit A (Table 4). The leucine 55 mutation has not been identified as a relevant mutation in other resistant Pseudomonas species, so it may be a novel mutation that aids in lowering ciprofloxacin's binding affinity therefore contributing to the acquisition of ciprofloxacin resistance. There was also a mutation in the topoisomerase IV gene in which the termination sequence was mutated to tyrosine (Table 4). The topoisomerase IV mutation function is unknown but may affect the structure of the enzyme leading to lower ciprofloxacin binding affinity.

Lastly, strain 3 gained imipenem resistance through the imipenem stressor. Upon acquisition of imipenem resistance by strain 3, strain 4 was developed. The previously identified mutations that were acquired under both neomycin and ciprofloxacin pressures were conserved in strain 4, so the bacteria were able to maintain these genetic changes (Table 4). The MdtABC-TolC multidrug efflux transport system was originally mutated in the $m d t C_{-} 4$ gene when the strain was exposed to neomycin (Table 4). After imipenem exposure, the strain retained these original mutations to the complex and gained another mutation to this complex, but in a different gene. A SNP was found in which alanine 546 was changed to proline in the $m d t C_{-} 1$ gene (Table 4). It is unknown what this exact mutation did to the protein and the transport system as a whole. However, an additional mutation to the MdtABC-TolC multidrug efflux transport system complex suggests that this efflux transporter plays a role in antibiotic resistance since the strain continued to mutate and maintain previous mutations to this efflux transporter as it adapted in response to the different antibiotic stressors. 
The MDR strain also acquired a mutation at the 78781 base pair position in which aspartic acid 617 was changed to glutamic acid, which lies within the $m r d A \_1$ gene (Table 4). This gene codes for penicillin-binding protein two, which plays a role in peptidoglycan biosynthesis. Imipenem's mechanism of action is to weaken the bacterial cell wall by binding penicillin-binding proteins to prevent peptidoglycan synthesis and weaken the cell wall. ${ }^{8}$ The mutation in the $m r d A \_1$ gene has not been an identified mutation in other imipenem resistant Pseudomonas species. However, mutations in this gene have been found in other bacterial strains that are resistant to the cell wall inhibitor class of drugs. ${ }^{16}$ Due to the nature of the mrdA_1 gene mutation in that both amino acids have similar structures and chemical properties, it cannot be concluded that this mutation was a key change that resulted in the gaining of imipenem resistance. It is plausible that the mutation may affect imipenem's ability to bind penicillinbinding protein two, leading to the resistance seen in strain 4. It is also possible that the combination of many mutations rather than the sole mrdA_1 gene mutation led to the resistance observed.

Many previous studies have addressed and identified key mutations in the acquisition of MDR in Pseudomonas species. In comparison to previous studies, many of the identified mutations appear to be novel in this study. Previous studies have found the MexXY multidrug efflux pump system to be a commonly expressed efflux pump in Psendomonas species as well as a commonly mutated efflux pump in other neomycin resistant Psendomonas species. ${ }^{17}$ While no mutations to the MexXY multidrug efflux pump were found in this study, mutations to other efflux pumps were identified. In response to ciprofloxacin, previous studies have found a variety of mutations that contribute to the acquisition of ciprofloxacin resistance in other Psendomonas species. The common mutations identified were mutations to the genes encoding the enzymes gyrase and topoisomerase IV. The mutation in the gyrB gene found in this study has been identified as a key mutation in the acquisition of resistance to ciprofloxacin in other Pseudomonas species by other studies. ${ }^{15}$ This result suggests the importance of the gene $g r y B$ in ciprofloxacin resistance. The other mutations to gyrase and topoisomerase IV enzymes that were identified by this study have not been previously identified, suggesting that the mutations may be novel. Lastly, in response to imipenem, previous studies have found mutations in the mrdA_1 gene. While there was a mutation identified in the $m r d A \_1$ gene by this study, the mutation found was not a previously identified mutation suggesting another potential novel mutation.

Antibiotics are widely used in both medical and agricultural industries. The overuse and abuse of these drugs have been linked to the emergence and persistence of antibiotic-resistant bacteria and the genes that facilitate such resistance. In this study, we evaluated an environmental microbe's ability to acquire resistance to multiple antibiotic drugs. Our data suggest that this environmental microbe could become resistant to not only one but four different types of antibiotic drugs under selective pressure. This phenomenon of acquiring resistance is not novel. However, this study highlights the importance of researching how resistance can be acquired in environmental rather than clinical microbes. As such microbes thrive in the environment, they also have the ability to pass their antibiotic-resistant genes to neighboring bacterial species through horizontal gene transfer. It is plausible to suggest that some of these environmental microbes are potential human pathogens and therefore, will be very difficult infections to treat with common antibiotic drugs. Antibiotic resistance is a worldwide public health crisis. Further studies must be conducted to determine the magnitude of selective pressure required for both clinical and environmental bacteria to acquire resistance and how pollution of antibiotic drugs in the environment can be mitigated.

\section{CONCLUSIONS}

The Pseudomonas isolate successfully gained and maintained resistance to neomycin, ciprofloxacin, and imipenem at initially lethal doses to the organism through a series of sequential exposures. These data provide evidence that the use of multiple antibiotics in local populations may contribute to MDR development. Through genome alignment and analysis, a variety of SNPs were identified between the original isolate and the MDR isolate. These SNPs suggest that efflux transporters and mutations in drug target proteins play vital roles in developing drug resistance.

\section{ACKNOWLEDGEMENTS}

Thank you to the College of St. Benedict St. John's University's for the support on my research endeavors. Additionally, the authors acknowledge the CSBSJU FDRC for funds supplied to D.M. A thanks to Sarah Rhodes for her contributions to the project. Additionally, thank you to Dr. Elyse Krautkramer for guidance and contributions to the project. Finally, we thank the technical work of Cristian Flores-Figueroa and Jeannette Munoz-Aguayo at the Mid-Central Research and Outreach Center. Bioinformatics were supported using tools available from the Minnesota Supercomputing Institute. Genome sequences are available from the authors (D.M.) upon request.

\section{REFERENCES}

1. Martinez, J. (2008) Antibiotics and Antibiotic Resistance Genes in Natural Environments, Science 18, 365-367. doi:10.1126/science. 1159483

2. Davies, J., and Davies, D. (2010) Origins and Evolution of Antibiotic Resistance, Mol Biol Rev 74, 417-433. doi:10.1128/MMBR.00016-10 
3. United States Department of Labor, Healthcare Wide Hazards: Multi-drug Resistant Organisms, https://www.osha.gov/SLTC/etools/hospital/ hazards/mro/mro.html (accessed May 2020)

4. Bonomo, R. (2006) Mechanisms of Multidrug Resistance in Acinetobacter Species and Pseudomonas aeruginosa, Clin Infect Dis 43, 49-56. doi:10.1086/504477

5. Munita, J., and Arias, C. (2016) Mechanisms of Antibiotic Resistance, Microbiol Spectr 4, 1-37. doi:10.1128/microbiolspec.VMBF0016-2015

6. CDC. (2019). Antibiotic Resistance Threats in the United States. Atlanta, GA: U.S. Department of Health and Human Services, CDC.

7. Hager, S., Jensen, E., Johnson, T., and Mitchell, D. (2017) Adaption of Escherichia coli to Antibiotic Cycling via Single Nucleotide Polymorphisms, Am J Undergrad Res 14, 17-34. doi:10.33697/ajur.2017.001

8. Van Hoek, A., Mevius, D., Guerra, B., Mullany, P., Roberts, A., and Aarts, H. (2011) Acquired antibiotic resistance genes: an overview, Front Microbiol 2, 1-27. doi:10.3389/fmicb.2011.00203

9. Du, D., Wang-Kan, X., Neuberger, A., Veen, H., Pos, K., Piddock, L., and Luisi, B. (2018) Multidrug efflux pumps: structure, function, and regulation, Nat Rev Microbiol 16, 523-539. doi:10.1038/s41579-018-0048-6

10. Mehta, R., Champney, S. (2003) Neomycin and Paromomycin Inhibit 30 S Ribosomal Subunit Assembly in Staphylococcus aureus, Curr Microbiol 47, 237-243. doi:10.1007/s00284-002-3945-9

11. Bankevich, A., Nurk, S., Antipov, D., Gurevich, A., Dvorkin, M., Kulikov, A., Lesin, V., Nikolenko, S., Pham, S., Prjibelski, A., Pyshkin, A., Sirotkin, A., Vyahhi, N., Tesler, G., Alekseyev, M., Pevzner, P. (2012) SPAdes: a new genome assembly algorithm and its applications to single-cell sequencing, J Comput Biol 19, 455-77. doi:10.1089/cmb.2012.0021

12. Seemann, T. (2014) Prokka: rapid prokaryotic genome annotation, Bioinformatics 30, 2068-9. doi:10.1093/bioinformatics/btu153

13. Darling, A., Mau, B., Blattner, F., Perna, N. (2004) Mauve: multiple alignment of conserved genomic sequence with rearrangements, Genome Res 14, 1394-1403. doi:10.1101/gr.2289704

14. Tikhonova, E., Dastidar, V., Rybenkov, V., and Zgurskaya, H. (2009) Kinetic control of TolC recruitment by multidrug efflux complexes, PNAS 106, 16416-16421. doi:10.1073/pnas.0906601106

15. Rehman, A., Patrick, W., and Lamont, I. (2018) Mechanisms of ciprofloxacin resistance in Pseudomonas aeruginosa: new approaches to an old problem, J Med Microbiol 68, 1-10. doi:10.1099/jmm.0.000873

16. Ranjitkar, S., Reck, F., Ke, X., Zhu, Q., McEnroe, G., Lopez, S., Dean, C. (2019) Identification of Mutations in the mrdA Gene Encoding PBP2 That Reduce Carbapenem and Diazabicyclooctane Susceptibility of Escherichia coli Clinical Isolates with Mutations in ftsI (PBP3) and Which Carry blaNDM-1, mSphere 4, 1-6. doi:10.1128/mSphere.00074-19

17. Chuanchuen, R., Wannaprasat, W., Ajarivakhajorn, K., Schweizer, H. (2008) Role of the MexXY multidrug efflux pump in moderate aminoglycoside resistance in Pseudomonas aeruginosa isolates from Pseudomonas mastitis, Microbiol Immunol 52, $392-398$. doi:10.1111/j.1348.0421.2008.00051.x

\section{ABOUT STUDENT AUTHOR}

Allison Grodnick graduated from the College of St. Benedict in St. Joseph, MN in May 2020. She earned a Bachelor's of Art with a major in Biochemistry. In the fall of 2021, she will begin graduate school at the University of Minnesota through their Biochemistry, Molecular Biology, and Biophysics Ph.D. program.

\section{PRESS SUMMARY}

As an unintentional result of the extensive use of antibiotics in healthcare and agriculture, antibiotics have become a prevalent selective pressure on bacteria. This pressure forces bacteria to evolve drug resistance through mutations in order to survive. This study aims to evaluate the acquisition of resistance to multiple antibiotics by an initially susceptible isolated bacterium from a Minnesota forest environment. Neomycin, ciprofloxacin, and imipenem were the selected antibiotics used to determine if this isolate could become resistant over time and exposure. The initial isolate acquired resistance to all three selected antibiotics. Single nucleotide polymorphisms between the original isolate and the final resistant strain were identified and are suspected to play a role in the isolate's gain of antibiotic resistance. Insight into how bacteria gain antibiotic resistance is essential in addressing this rising health issue. 